\title{
Is Buddy Taping as Effective as Plaster Immobilization for Adults With an Uncomplicated Neck of Fifth Metacarpal Fracture? A Randomized Controlled Trial
}

\author{
Richard Pellatt, BA, MBChB*; Igor Fomin, BA, BN; Carli Pienaar, BSC; Randipsingh Bindra, FAAOS, MCh Ortho; \\ Michael Thomas, MBChB, FRACS (Ortho); Ezekiel Tan, MBChB, FRACS (Ortho); Cindy Mervin, BSC; Ping Zhang, BSC, PhD; \\ Gerben Keijzers, MBBS, PhD \\ *Corresponding Author. E-mail: drpellatt@googlemail.com or richard.pellatt@health.qld.gov.au.
}

\begin{abstract}
Study objective: We compare buddy taping with plaster casting for uncomplicated fifth metacarpal (boxer's) fractures. We hypothesize buddy taping will give superior functional outcomes at 12 weeks, defined as a 10-point difference on the Shortened Disabilities of the Arm, Shoulder and Hand (quickDASH) score.
\end{abstract}

\begin{abstract}
Methods: This randomized controlled trial included patients aged 18 to 70 years, with uncomplicated boxer's fractures in 2 hospitals in Queensland, Australia. The intervention consisted of buddy taping of the ring and little fingers on the affected side, in which the control group received plaster casting. Primary outcome was hand function as measured by quickDASH score (0 to 100 , with 0 indicating no disability) at 12 weeks. Secondary outcomes measured at 3, 6, and 12 weeks included time off work and activities, pain, satisfaction, and the EuroQol 5-Dimension 3-Level score (measure of overall health).
\end{abstract}

Results: Ninety-seven patients with primary endpoint data were available for analysis, 48 in the buddy taping group and 49 in the plaster group. At 12 weeks, median quickDASH scores were the same for both groups (buddy 0, interquartile range [IQR] 0 to 2.3; plaster 0, IQR 0 to 4; difference 0; 95\% confidence interval of the difference 0 to 0). Patients in the buddy taping group missed a median 0 days (IQR 0 to 7) of work compared with the plaster group's 2 days (IQR 0 to 14). Other secondary outcome measures were the same in both groups.

Conclusion: We found that patients with boxer's fractures who were randomized to buddy taping had functional outcomes similar to those of patients randomized to plaster cast at 12 weeks. We advocate a minimal intervention such as buddy taping for uncomplicated boxer's fractures. [Ann Emerg Med. 2019; $\mathbf{a : 1 - 1 0 . ] ~}$

Please see page XX for the Editor's Capsule Summary of this article.

\section{INTRODUCTION}

\section{Background}

Fractures of the fifth metacarpal neck-boxer's fractures-are common, accounting for $20 \%$ of hand injuries $^{1}$ and $5 \%$ of fractures of the upper extremity. ${ }^{2}$ They are usually the result of a closed fist strike and are associated with a young, working-age-male demographic. ${ }^{3,4}$ As such, boxer's fractures can have a significant functional effect and result in time off work, leading to wider social and economic effects. ${ }^{5}$

Uncomplicated boxer's fractures-minimally displaced, closed, isolated injuries, with a fracture angulation up to 70 degrees-are managed conservatively without surgery. ${ }^{6,7}$ A range of options exists, such as immobilization in a cast, buddy taping of the ring and little fingers, and functional strapping of the affected hand. ${ }^{2}$

A 2005 Cochrane review found that existing studies comparing interventions for boxer's fractures were underpowered to detect a difference in functional outcomes, and could not clearly recommend one modality over another. ${ }^{2}$ In more recent randomized controlled trials and meta-analyses, functional outcomes were similar., ${ }^{9,10,14}$ Because discomfort, hand function, and ability to work may be considered variably per patient-clinician interaction, the current data are insufficient to provide consensus for the optimal management of patients with boxer's fractures. ${ }^{2,9}$ 


\section{Editor's Capsule Summary}

What is already known on this topic

Plaster immobilization is a standard emergency department (ED) treatment for patients with a fifth metacarpal (boxer's) fracture, but buddy taping is less studied.

What question this study addressed

This randomized, nonblinded trial compared plaster casting with buddy taping in ED patients with a boxer's fracture. Primary outcome measure was hand function at 12 weeks, measured by the Shortened Disabilities of the Arm, Shoulder and Hand score.

What this study adds to our knowledge

Functional outcomes were similar at 12 weeks.

How this is relevant to clinical practice

Buddy taping may be a reasonable treatment option for selected ED patients with an uncomplicated boxer's fracture.

\section{Importance}

Management options for boxer's fractures can affect a patient's ability to return to work, sports, and hobbies. ${ }^{15}$ Plaster casts immobilize the affected joint, which might lead to better anatomic healing and thus outcome. ${ }^{6}$ However, a cast can necessitate time off work, or at least a modification to duties. ${ }^{12}$ Comparatively, functional strapping or taping allows early mobilization and as such might allow earlier return to work and improved hand function. ${ }^{10-12}$

Existing literature has either been underpowered or unable to detect a difference in patient functional outcomes or has concentrated on radiologic and physical measurements. ${ }^{2}$ Functional outcomes are patient centered and arguably more important than surrogate outcomes. Health economic implications of management options have not been explored in studies thus far, to our knowledge. ${ }^{15}$

The demographic associated with boxer's fractures is not always compliant with follow-up, as evidenced in previous studies. ${ }^{10,16}$ A less cumbersome intervention, such as functional taping, might benefit the patient and reduce the need for orthopedic follow-up, requiring fewer radiographic and specialist reviews. ${ }^{16,17}$ Outcomes at a patient, hospital, and community level might be significantly influenced by management choice.

To date, to our knowledge no randomized controlled trial or broader review has identified a superior treatment considering both hand function and resource use. ${ }^{15} \mathrm{We}$ hypothesized that buddy taping would provide better functional outcomes than plaster casting and might allow an earlier return to work.

\section{Goals of This Investigation}

The objective of this study was to assess whether functional (buddy) taping of the ring and little fingers showed superior functional outcomes at 12 weeks compared with plaster immobilization in the management of adult patients with boxer's fractures. Primary outcome was hand function as measured by the Shortened Disabilities of the Arm, Shoulder and Hand (quickDASH) questionnaire 12 weeks postinjury. Prespecified secondary outcomes included patient-reported pain and satisfaction scores, time missed from work and sports, and health economic outcomes.

\section{MATERIALS AND METHODS}

\section{Study Design and Setting}

This was a randomized controlled trial conducted between March 2016 and December 2017 in the emergency departments (EDs) of 2 public hospitals within the same health district in Southeast Queensland, Australia. The main campus is a 750-bed major metropolitan teaching hospital and the second campus, located $12 \mathrm{~km}$ away, is an urban district hospital with 200 beds. The combined ED census in 2016 was 160,000 patients.

The study was designed and endorsed by emergency and orthopedic specialists working at both hospitals. The study was approved by the Health District's Human Research and Ethics Committee and registered before commencement. We adhered to the Consolidated Standards of Reporting Trials statement (http://www. consort-statement.org).

\section{Selection of Participants}

Patients were considered for the study if they had a suspected fifth metacarpal neck (boxer's) fracture. To be eligible for inclusion, a fracture needed to be confirmed on radiographs taken in 2 planes of view according to hospital protocol and guidelines. Patients were screened 24 hours a day, 7 days a week.

Exclusion criteria were younger than 18 years or older than 70 years; open fracture; gross rotational deformity of the affected finger as judged by the treating clinical team; comminuted fracture; intra-articular fracture; associated tendon injury, polytrauma, or other significant injury at presentation; fracture angulation greater than 70 degrees; and injury older than 1 week at presentation. The degree of accepted angulation was based on existing literature ${ }^{6}$ and 
after consultation with and endorsement by the institution's orthopedic team.

Eligible patients were provided with an information leaflet about the study. Written informed consent was obtained from the patient before enrollment. Patients were enrolled by the treating physician, nurse practitioner, or extended scope physiotherapist.

We randomized patients by using computer-generated block randomizations, with blocks of 10 for both hospitals. Sealed study packs were created with sequentially numbered opaque envelopes. Within each pack, a smaller, sealed opaque envelope contained the study identification and randomization arm.

Study packs were located in a designated display rack in the minor injuries areas of the EDs at both sites. Completed packs were placed in a sealed box next to the study packs. Once a suitable patient had consented, the next sequentially numbered envelope was opened to reveal the allocated treatment arm.

\section{Interventions}

Patients were randomized to receive either buddy taping of the ring and little fingers or plaster cast immobilization. The intervention arm involved buddy taping of the ring and little fingers of the affected hand, allowing movement of the wrist and hand (Figure 1). The ring and little fingers were taped together at the proximal and middle phalanges. The control arm, the hospital standard practice, involved cast immobilization in an ulnar gutter cast applied in a position of safe immobilization (Figure 2).

Buddy tape and plaster were applied by a designated plaster technician or the treating clinician when no plaster technician was available. All clinicians had received training in both buddy tape and plaster application. This is routine practice at both study sites.

In both groups, patients were instructed to have the allocated treatment (either buddy taping or plaster) applied for 3 weeks, and that the allocated treatment would be reviewed in the fracture clinic at designated follow-up times.

Patients in both groups were advised they could return to work when able, with no prespecified time off. Patients were given buddy tape and plaster care instructions, including advice to return to the ED if they had concerns about the applied treatment.

In both study arms, treating clinicians were instructed that fracture angle reduction was not required before the application of the allocated treatment. Blinding of the treating clinician and patient was, by virtue of the interventions involved, not possible. The statistician was blinded to group allocation. Patients were followed up by

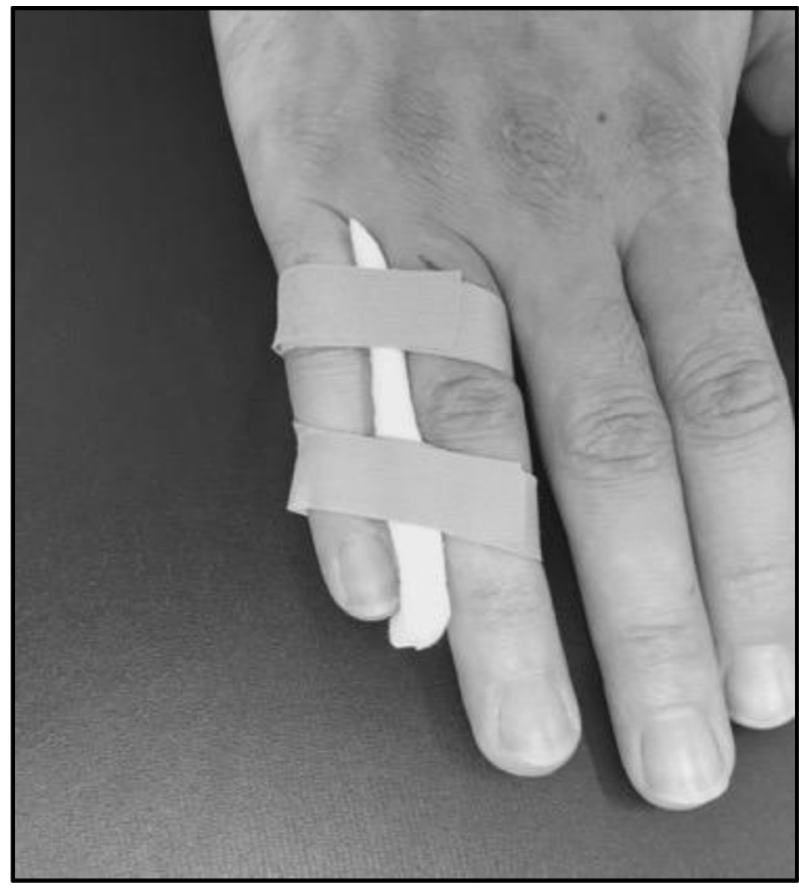

Figure 1. Buddy strapping.

the research team and a dedicated research nurse. Patients were followed up in the clinic, and if they did not present for follow-up, they were contacted by telephone.

\section{Outcome Measures}

The primary outcome was hand function at 12 weeks, measured with the validated quickDASH questionnaire, ${ }^{18}$ which reflects the patient's ability to do everyday tasks and measures pain and disability as a result of the injury. The quickDASH questionnaire scores a patient's overall disability on a scale of 0 to 100 , calculated from 11 questions. Higher scores equate to a higher degree of disability. The measure has been used previously when functional outcomes of boxer's fractures have been assessed ${ }^{10}$ and has been validated for use in person, as well as over the telephone. ${ }^{19,20}$ quickDASH scores were measured at 12 weeks as the primary outcome and compared between the 2 groups. When possible, the scores were measured at 3 and 6 weeks to allow imputation at final analysis in case of possible loss to follow-up at 12 weeks. The optional work and sports-performing arts modules of the quickDASH questionnaire were not included.

Secondary outcome measures included pain and satisfaction according to a numeric rating score from zero to 10 , in which zero equated to no pain and 10 to severe pain, and in which zero equated to very unsatisfied and 10 to fully satisfied; return to work (days off); return to sports 

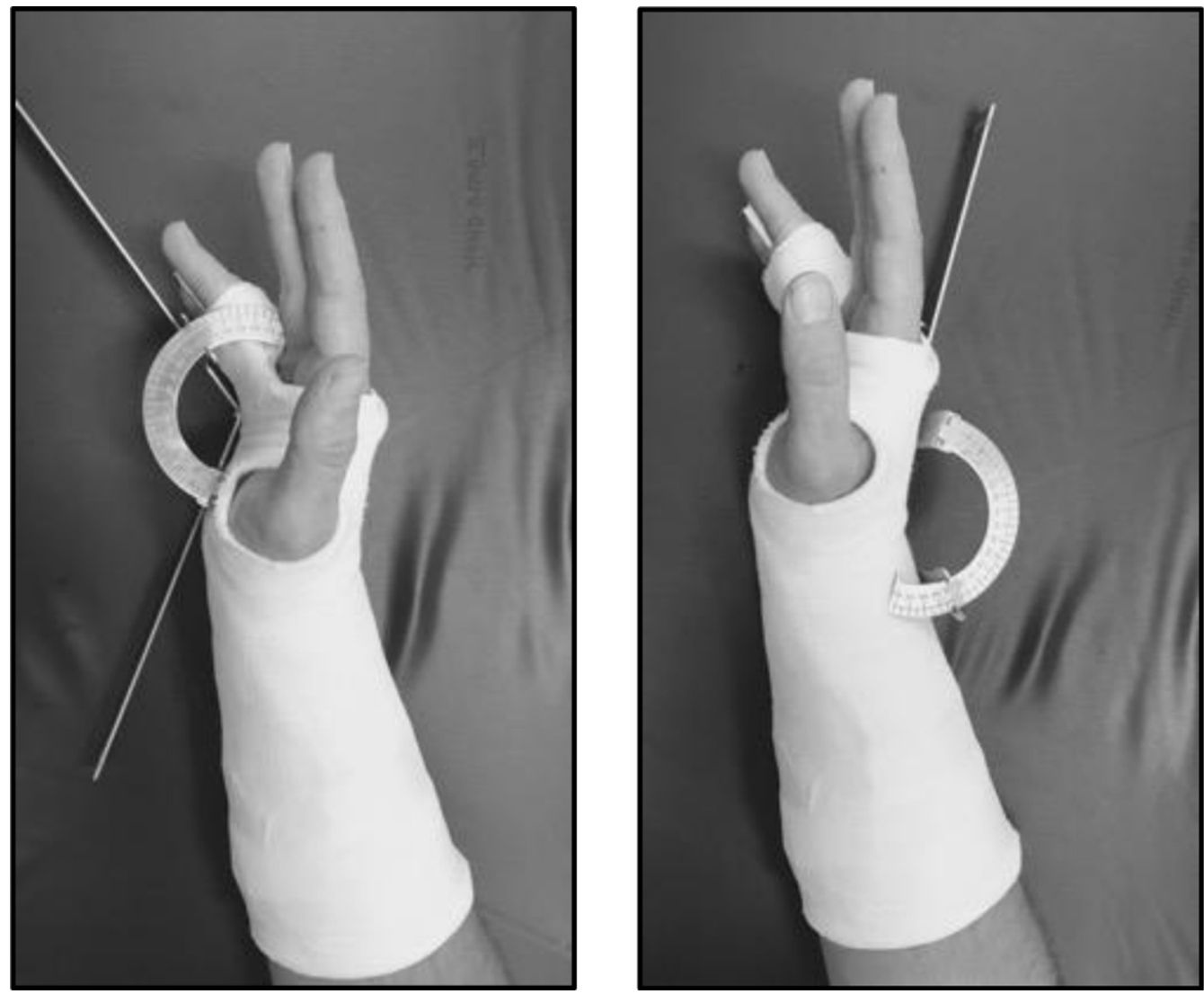

Figure 2. Cast immobilization in a position of safe immobilization.

(days off); and a validated measure of overall health-related quality of life, the EuroQol 5-Dimension 3-Level (EQ-5D3L) questionnaire. ${ }^{21}$ The EQ-5D-3L questionnaire asks patients to estimate their health on a scale of 1 to 3 ( 1 being good health, 3 being poor health) in terms of mobility, selfcare, usual activities, pain or discomfort, and anxiety or depression, generating a score between 5 and 15 , with a lower score indicating better overall health. EQ-5D-3L scores were calculated at presentation and at 12 weeks. ${ }^{22}$ Pain and satisfaction were measured at weeks 1 and 12 .

Medicare is the publicly funded universal health care system in Australia, and as such there were no direct monetary costs for the patients enrolled in this study. Health care resource utilization data were collected for the 2 study groups. These resources included equipment cost (buddy taping and plaster) and staff time. The cost of materials used for a plaster cast is estimated at AUD \$22 compared with AUD $\$ 2$ for the cost of buddy taping. The cost of a plaster technician is estimated at AUD $\$ 33 / \mathrm{h}$.

\section{Primary Data Analysis}

The sample size calculated for the study was 98 patients, 49 in each arm. The sample size was based on the ability to detect a clinically significant difference in the quickDASH score (from 0 to 100) of 10 points with an SD of 20 points at 12 weeks, with a power of $80 \%$ and a 2 -sided $\alpha$ of .05. A minimal clinically important difference of 10 points was based on existing recent literature. ${ }^{10}$

Analysis was by intention to treat. Patients with available outcome data were analyzed according to group assignment. Additional sensitivity analyses, including imputations and per-protocol analysis, were conducted. Imputation analysis involved using data from the last available follow-up if primary outcome data at week 12 were not available. Per-protocol analysis involved analyzing the patients by their final allocation, rather than the group they were randomized to.

Data were entered into an Excel (version 16.21.1; Microsoft, Redmond, WA) spreadsheet and then analyzed in R (version 3.4.2; R Development Core Team, Auckland, New Zealand). Before analysis, variables were reviewed for accuracy of data entry, missing values, and outliers. We report medians, interquartile range (IQR), and 95\% confidence intervals (CIs) as appropriate.

Prespecified secondary health economic outcomes were reported from the perspective of Queensland Health, Australia. All costs are reported in 2016 Australian dollars (AUD \$1 equals approximately US \$0.75). 


\section{RESULTS}

\section{Characteristics of Study Subjects}

Five hundred six patients with presenting complaints suggestive of boxer's fractures were considered for inclusion, with Figure 3 outlining reasons for noninclusion. One hundred twenty-six patients were randomized and 97 patients with primary endpoint were available for intention-to-treat analysis (Figure 3).
Baseline demographics of the study population are described in Table 1. Patients were predominantly young (26.5 years), men (85\%), and right-hand dominant $(90 \%)$. The most common employment type in both the buddy tape and plaster groups was technical or trade (35\% versus $39 \%$ ), and in both groups approximately one third played a sport as their main hobby.

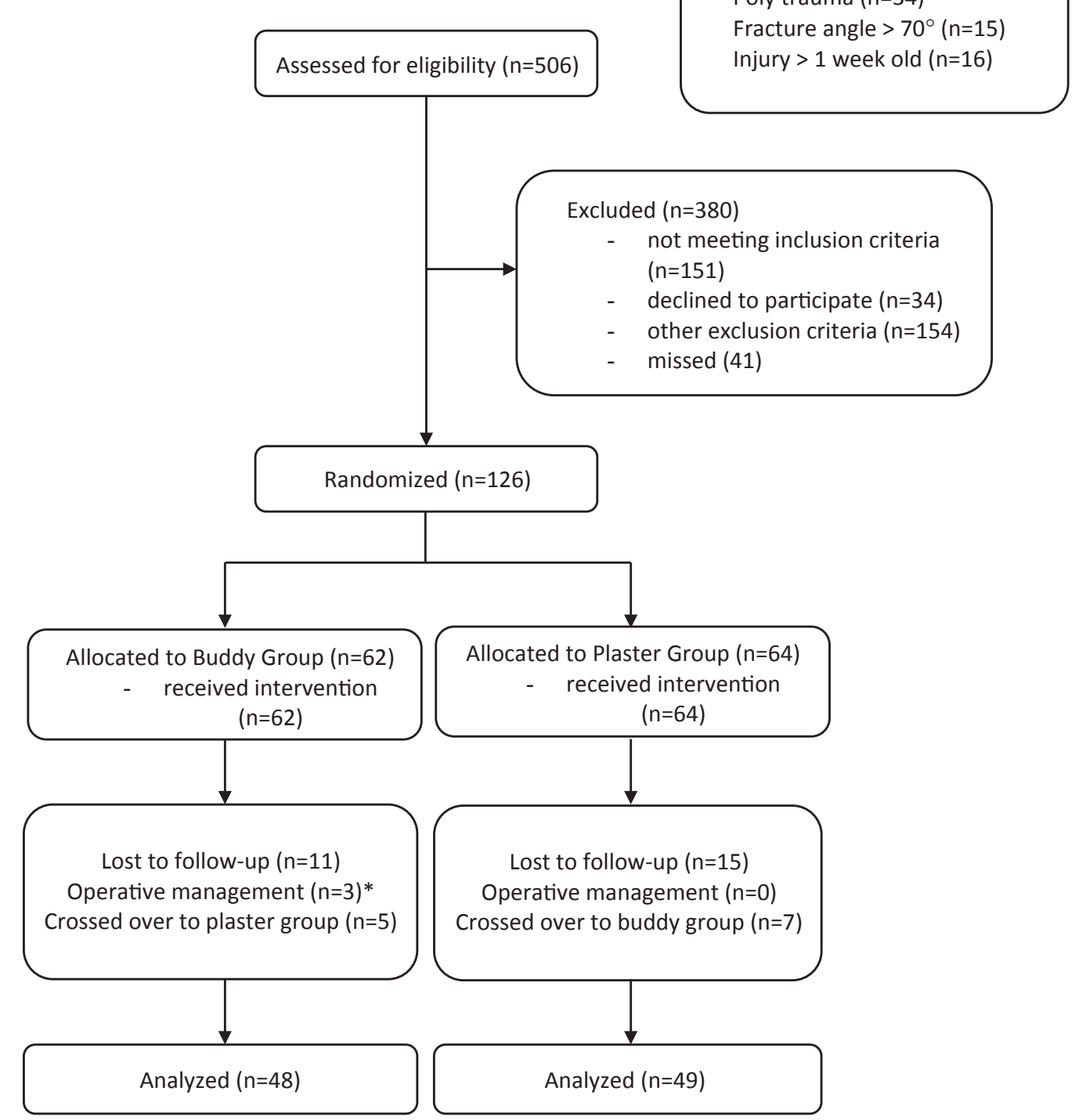

Exclusion Criteria ( $n=154)$

Age $<18(n=50)$

Open fracture $(n=2)$

Rotational deformity $(n=11)$

Comminuted fracture $(n=15)$

Intraarticular fracture $(n=11)$

Tendon injury $(n=0)$

Poly trauma $(n=34)$

Fracture angle $>70^{\circ}(n=15$

Injury $>1$ week old $(n=16)$

not meeting inclusion criteria $(n=151)$

declined to participate $(n=34)$

other exclusion criteria $(n=154)$

missed (41)

Figure 3. Consolidated Standards of Reporting Trials flow diagram. *Patients undergoing operative management were excluded from the final analysis. 


\section{Main Results}

At 12 weeks, median quickDASH scores were the same for both groups (buddy 0, IQR 0 to 2.3; plaster 0 , IQR 0 to 4; difference 0 ; $95 \%$ CI of difference 0 to 0 ) (Figure 4). Patients randomized to the buddy group returned to work earlier than those randomized to the plaster group. Patients with buddy taping missed no days of work (IQR 0 to 7) compared with those immobilized in a plaster cast, who missed a median average of 2 days of work (IQR 0 to 14). There was no difference in days missed from hobbies, activities, and sports between the 2 groups (buddy median 30 days, IQR 23 to 35 days; plaster median 35 days, IQR 0 to 41 days) (Table 2). There was no difference in EQ-5D-3L score between the 2 groups at 12 weeks (Table 2). Patients had similar pain and satisfaction scores at 1 and 12 weeks (Table 2), with patients in both groups reporting absence of pain and high satisfaction with treatment.

Table 1. Baseline demographics.

\begin{tabular}{|c|c|c|}
\hline Demographics & Buddy $(n=62)$ & Plaster $(n=64)$ \\
\hline \multicolumn{3}{|l|}{ Week 0} \\
\hline Age, median (IQR), y & $26(20-38)$ & $27(20-34)$ \\
\hline Male sex, No. (\%) & $51(82.2)$ & $56(87.5)$ \\
\hline Left-handed, No. (\%) & $6(9.7)$ & $6(9.4)$ \\
\hline \multicolumn{3}{|l|}{ Occupation, No. (\%) } \\
\hline Manager & $4(6)$ & $6(9)$ \\
\hline Professional & $10(16)$ & $5(8)$ \\
\hline Technical and trade & $22(35)$ & 25 (39) \\
\hline $\begin{array}{l}\text { Community and personal } \\
\text { services }\end{array}$ & $9(15)$ & $14(22)$ \\
\hline Clerical and administrative & $1(2)$ & $2(3)$ \\
\hline Sales & $4(6)$ & $2(3)$ \\
\hline Machinery operators and drivers & $3(5)$ & $1(2)$ \\
\hline Unemployed & $9(15)$ & $9(14)$ \\
\hline \multicolumn{3}{|l|}{$\begin{array}{l}\text { Hobbies, activities, and sports } \\
\text { class, No. (\%) }\end{array}$} \\
\hline Sport & $25(40)$ & $24(37.5)$ \\
\hline Social & 0 & $1(2)$ \\
\hline Arts & 0 & 0 \\
\hline Outdoors* & $8(13)$ & $15(23)$ \\
\hline None & $29(47)$ & $24(37.5)$ \\
\hline EQ-5D-3L score, median (IQR) & $5(5-6)$ & $5(5-6)$ \\
\hline $\begin{array}{l}\text { Premorbid quickDASH score, } \\
\text { median (IQR) }\end{array}$ & $0(0-11.36)$ & $6.8(0-22.7)$ \\
\hline $\begin{array}{l}\text { Fracture angle at presentation, } \\
\text { median (IQR), degrees }\end{array}$ & $28.7(10-40)$ & $30.8(16-43)$ \\
\hline
\end{tabular}

*Outdoors included activities such as walking, hiking, gardening, and fishing.
Three patients (all randomized to the buddy group) underwent operative management. In the first 2 cases, at the fracture clinic, the treating orthopedic consultant thought the injuries were intra-articular, and a shared decision with patients led to operative management. In the third case, the patient reinjured the affected limb at 6 weeks, received a plaster cast, and underwent operative management. Five patients in the buddy group received a plaster cast instead, 4 at first fracture clinic follow-up; 1 patient who re-presented to the ED 2 days after randomization requested a cast. Seven patients in the plaster group were switched to buddy strapping at the first follow-up appointment (Figure 3). In both groups, crossover was a combination of patient and treating clinician preference.

When possible, repeated quickDASH scores were recorded at 3 and 6 weeks; overall score was higher than at 12 weeks for both groups, with a sequential reduction in median score over time (Figure 4). Sensitivity analyses were undertaken with analysis by imputation, as well as per protocol, analyzed by the final treatment arm patients received, which included those who crossed over. In imputation analysis, patients lost to follow-up at 12 weeks were included, using their last available data, at week 1, 3, or 6, carried forward. A total of 109 patients were analyzed in the imputation analysis, with 54 in the buddy group and 55 in the plaster group. Neither analysis affected overall results.

Median length of stay in the ED was shorter by greater than half an hour for patients randomized to the buddy group compared with the plaster group (buddy group 140 minutes, IQR 98 to $201,95 \%$ CI 116 to 160 ; plaster group 176 minutes, IQR 123 to 236, 95\% CI 141 to 193; difference 36 minutes).

Of the patients who attended follow-up appointments and received repeated radiographs, no complications (eg, infection, nonunion, delayed union) were reported. Fracture angle at follow-up was similar for both groups (median 32.6 degrees for the buddy group [IQR 20 to 44] and 28.1 degrees for the plaster group [IQR 10 to 38.65]).

\section{LIMITATIONS}

There are several limitations to this study. It was designed as a superiority study; however, based on the assumption that buddy taping would have given the same results with less resource use, a noninferiority design would have been preferable. A post hoc sample size calculation for a noninferiority design showed that based on a noninferiority margin of 10 points on the quickDASH questionnaire (the same as used in previous research ${ }^{10}$ ), an 


\section{QuickDASH Scores}
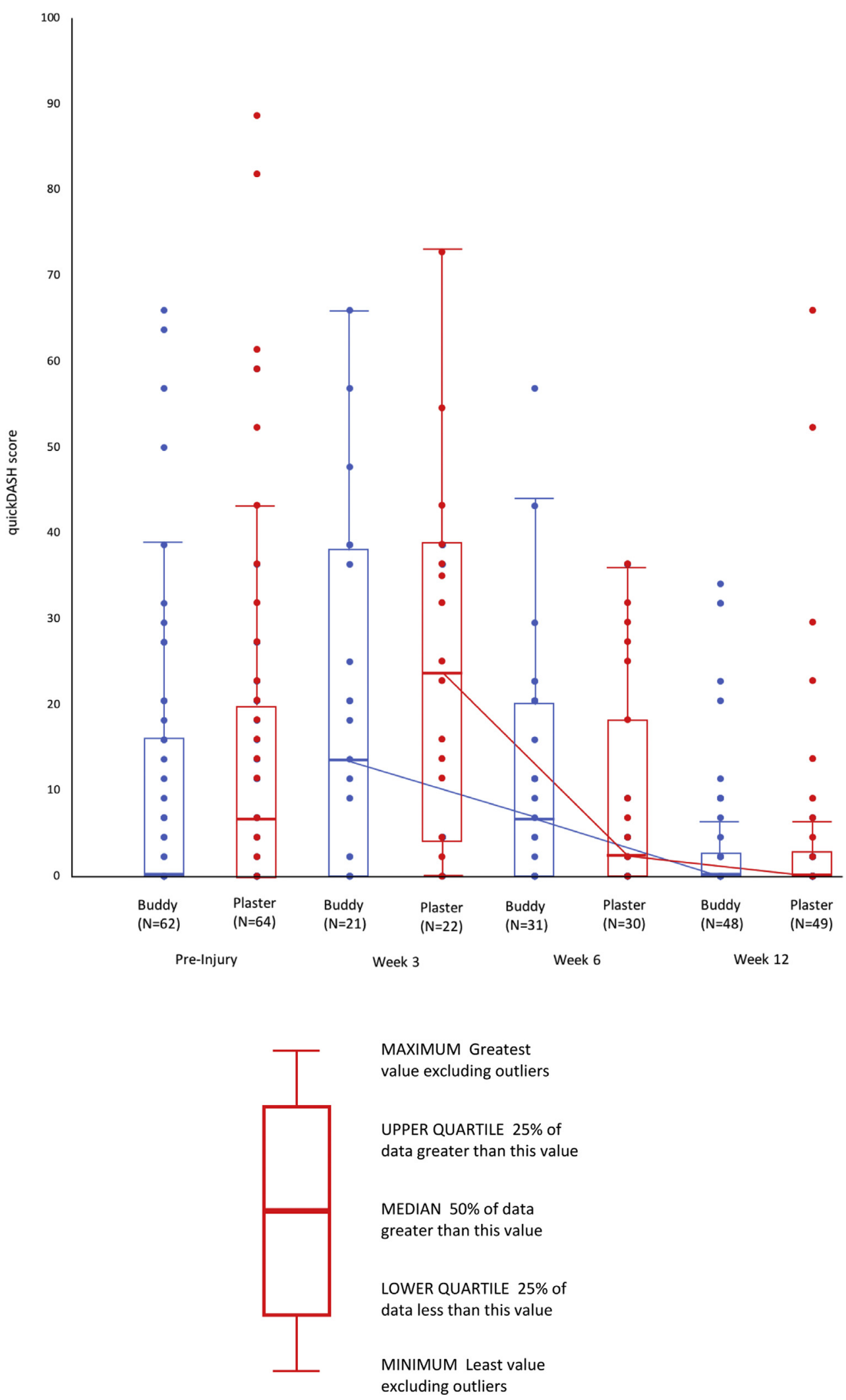

Figure 4. quickDASH scores over time. 
Table 2. Secondary outcome measures.

\begin{tabular}{|c|c|c|c|c|}
\hline Secondary Outcome Measures & $\begin{array}{l}\text { Buddy Group } \\
\quad(n=48)\end{array}$ & $\begin{array}{l}\text { Plaster Group } \\
\quad(n=49)\end{array}$ & $\begin{array}{c}\text { Difference Between Groups } \\
\text { ( } \Delta=\text { Buddy-Plaster) }\end{array}$ & $95 \% \mathrm{Cl}$ of $\Delta$ \\
\hline EQ-5D-3L score at wk 12 , median (IQR)* & 5 (5 to 6$)$ & 5 (5 to 6$)$ & 0 & 0 to 0 \\
\hline Pain score at wk 1 , median $(\mathrm{IQR})^{\dagger}$ & 0 (0 to3) & 0 (0 to3) & 0 & 0 to 0 \\
\hline Pain score at wk 12, median (IQR) & $0(0$ to 0$)$ & 0 (0 to 1$)$ & 0 & 0 to 0 \\
\hline Satisfaction score at wk 1 , median $(\mathrm{IQR})^{\ddagger}$ & 9 (7 to 10$)$ & 9 (8 to10) & 0 & -1 to 1 \\
\hline Satisfaction score at wk 12, median (IQR) & $9(8$ to 10$)$ & 9 (8 to 10$)$ & 0 & 0 to 1 \\
\hline Missed work, median (IQR), days & $0(0$ to 7$)$ & $2(0$ to 14$)$ & 1 & 0 to 3 \\
\hline Missed hobbies and sports, median (IQR), days & 30 (23 to 35$)$ & $35(0$ to 41$)$ & 0 & -8 to 12 \\
\hline 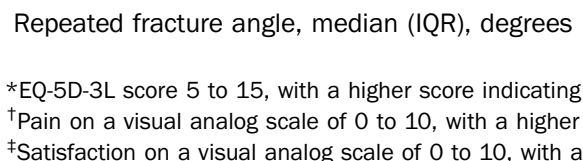 & $\begin{array}{l}32.6 \text { (20 to } 44) \\
\text { health. } \\
\text { indicating worse pa } \\
\text { r score indicating gr }\end{array}$ & 28.1 (10 to 38.65 ) & 5 & -2.4 to 14.9 \\
\hline
\end{tabular}

SD of 10 points, and a $90 \%$ power, a sample size of 21 per group would have been required, suggesting that despite the superiority design, our sample would have supported a noninferiority conclusion.

Forty-one potentially eligible patients were not considered for inclusion by the treating clinician, and 34 patients declined to participate (Figure 3). Patients were missed because of other clinical priorities in a busy workplace environment. These potentially eligible patients had demographics similar to those of the patients who were included, making selection bias unlikely.

The quickDASH score asks patients to report hand function related to the 7 days before. We measured the baseline (preinjury) quickDASH score to identify patients with preexisting decreased function. High preinjury quickDASH scores were reported at baseline in both groups. However, because most patients had good functional outcomes at 12 weeks, this suggests that some patients may have erroneously reported their postinjury function, accounting for high baseline scores. There was no statistically significant difference in baseline quickDASH scores between the groups, indicating this occurred in a similar manner in both groups. Equally, other baseline demographics were evenly distributed, and as such a baseline imbalance in function is unlikely, leaving the primary outcome still valid. We suggest that future research ask specifically about both pre- and postinjury quickDASH scores at baseline.

Because of logistic barriers during fracture clinic followup, we were unable to obtain objective measurements of grip strength and range of motion, something that was intended in the study protocol. When patients did attend follow-up clinic, repeated radiographs and fracture angle measurements were taken as planned. Because our functional outcome was similar between groups, we believe that grip strength and range of motion were unlikely to vary greatly between groups.

When patients failed to attend follow-up in person, quickDASH assessments occurred by telephone. This was anticipated in the study protocol, and verbal quickDASH assessments have been previously reported to be reliable and valid. ${ }^{19,20}$ Although we cannot exclude the potential for inaccuracies in telephone assessments, such an effect would have been similar for both groups.

Seven patients in the plaster group and 5 in the buddy group crossed over to the alternative treatment arm. In each case, this was a combination of patient request and clinician discretion. Our primary analysis was intention to treat, although we tested robustness of our findings with imputation and per-protocol analysis, which found similar results.

Three patients in the buddy taping group underwent operative management. For one patient, this was due to a repeated injury. The other 2 patients underwent operative management at the discretion of the orthopedic clinician, who believed the injuries to be intra-articular. We are unable to comment on whether these decisions were related to treatment allocation.

Several secondary outcomes, including variables of a health economic nature, such as days off work, time spent in the $\mathrm{ED}$, and unit cost of treatment, were measured; however, a formal cost analysis was not conducted. The study was not powered to show differences in secondary outcomes.

Last, study participants, treating clinicians, and researchers were unable to be blinded to allocation by virtue of the nature of the intervention. Although some secondary outcomes may have been affected by this, we believe it is unlikely primary outcome assessment would be affected because at 12 weeks neither group had any form of immobilization in place. 


\section{DISCUSSION}

Patients in both the buddy taping and plaster groups had the same good functional outcomes, as measured with the quickDASH questionnaire. Patients randomized to the buddy taping group returned to work earlier than those randomized to the plaster group. Pain and satisfaction scores were similar for both groups at 1 and 12 weeks. There was no difference in overall quality of life according to EQ-5D-3L scores between the 2 groups at 12 weeks. Of patients who attended follow-up clinic appointments and received follow-up radiographs, there were no complications in either group, and healed fracture angles were similar. Patients randomized to the buddy taping group spent, on average, 36 minutes less time in the ED than those randomized to the plaster group.

The results show that for patients with boxer's fractures, buddy taping resulted in outcomes similar to those of the plaster casting in regard to hand function and other prespecified secondary outcome measures at 12 weeks. Patients randomized to the buddy taping group missed less time from work and were able to return to predominantly trade and labor roles that a plaster cast might negate performing.

These outcomes have implications for patient follow-up. With no discernible influence of buddy taping on fracture healing and function, patients with that treatment could be followed up in community settings such as family practice clinics, rather than in hospital orthopedic clinics, as is current practice in our hospital for patients with plaster casts.

Patients randomized to buddy taping spent less time in the ED than those in the plaster group. We postulate that this was because buddy taping is quick and easy to apply and does not require the expertise of a plaster technician. This has benefits for both EDs and orthopedic departments. Clinicians in the ED can treat patients quickly and effectively, whereas plaster technicians can remain dedicated to other injures either in the ED or orthopedic fracture clinic.

Patients in our study returned to work earlier than those in previous studies. For the buddy group, we postulate the earlier return to work (median time missed 0 days compared with 22 days in one previous study ${ }^{10}$ ) may have been due to a tendency among clinicians to encourage patients to return to work when able. For the plaster group, we expected patients to have taken more than 2 days off because of the restriction of a cast. Indeed, in one previous study, patients missed on average a month of work. ${ }^{10}$ It is conceivable that patients in the plaster group returned to work on lighter duties, and future research could establish whether this is the case. Alternatively, telephone follow-up may have introduced recall bias.

A plaster cast is usually applied by a trained plaster technician at $\$ 33 / \mathrm{h}$, and the cost of materials required for buddy taping is cheaper than that for plaster casting (buddy taping less than AUD \$2 and plaster cast AUD \$20). Buddy taping can be applied directly by the treating clinician, with no extra specialist service. Hence, buddy taping represents a unit material cost saving and reduces ED resources.

Our study demographic is comparable to patient demographics in previous studies, and thus we believe our results are generalizable to other high-income countries. The suboptimal rate of clinic follow-up observed in previous studies was also reflected in our own. ${ }^{10,16}$ However, the study design allowed follow-up to be conducted by telephone, resulting in higher follow-up rates at time frames similar to those reported in previous literature. ${ }^{10}$ Because hand function at 12 weeks was reported as optimal by patients in both groups, this likely would be sustained for the longer term.

In accordance with the patient-centered outcomes of our trial, and in context of other published evidence, we recommend a minimal intervention such as buddy taping for the management of uncomplicated boxer's fractures. ${ }^{15}$ Such an intervention has functional outcomes for the patient similar to those of plaster immobilization, seems to allow an earlier return to work, and saves resources in both the ED and orthopedic department.

The authors acknowledge Paul Scuffham, PhD, for providing health economics consulting, Martin Smith, $M B C h B$, for aiding with data collection, Deborah Lenaghan, $M A$, for providing study design consulting, and Amy Sweeny, $M A$, for providing consulting regarding patient follow-up.

Supervising editor: Gregory W. Hendey, MD. Specific detailed information about possible conflict of interest for individual editors is available at https://www.annemergmed.com/editors.

Author affiliations: From Gold Coast University Hospital (Pellatt, Bindra, Thomas, Tan, Keijzers); Griffith University (Fomin, Pienaar, Bindra, Mervin, Zhang, Keijzers), Southport, Australia; and Bond University, Robina, Australia (Keijzers).

Author contributions: RP and GK conceived the study. RP, RB, MT, ET, and GK were involved in the study design. RP and GK obtained research funding. RP, IF, and CP undertook recruitment of patients. PZ provided statistical advice and analyzed the data. CM provided health economic analysis. RP drafted the article and all authors substantially contributed to revisions. RP takes responsibility for the paper as a whole.

All authors attest to meeting the four ICMJE.org authorship criteria: (1) Substantial contributions to the conception or design of the 
work; or the acquisition, analysis, or interpretation of data for the work; AND (2) Drafting the work or revising it critically for important intellectual content; AND (3) Final approval of the version to be published; AND (4) Agreement to be accountable for all aspects of the work in ensuring that questions related to the accuracy or integrity of any part of the work are appropriately investigated and resolved.

Funding and support: By Annals policy, all authors are required to disclose any and all commercial, financial, and other relationships in any way related to the subject of this article as per ICMJE conflict of interest guidelines (see www.icmje.org). This research was funded by a grant from the Emergency Medicine Foundation, Australia. Dr. Bindra reports consulting for Acumed LCC, Integra LifeSciences LCC, and Actelion.

Publication dates: Received for publication July 6, 2018.

Revisions received October 27, 2018, and January 5, 2019. Accepted for publication January 17, 2019.

Presented at the Australasian College of Emergency Medicine annual scientific meeting, November 2017, Sydney, Australia; and the Gold Coast University Hospital Research Week conference, November 2017, Gold Coast, Australia.

Trial registration number: ACTRN12616000441415

\section{REFERENCES}

1. Keenan M. Managing boxer's fracture: a literature review. Emerg Nurse. 2013;21(16):18-24.

2. Poolman RW, Goslings JC, Lee JB, et al. Conservative treatment for closed fifth (small finger) metacarpal neck fractures. Cochrane Database Syst Rev. 2005;3:CD003210.

3. Greer SE, Williams JM. Boxer's fracture: an indicator of intentional and recurrent injury. Am J Emerg Med. 1999;17: 357-360.

4. Soong M, Got C, Katarincic J. Ring and little finger metacarpal fractures: mechanisms, locations, and radiographic parameters. J Hand Surg Am. 2010;35:1256-1259.

5. Gudmundsen TE, Borgen L. Fractures of the fifth metacarpal. Acta Radiol. 2009;50:296-300.

6. Statius Muller MG, Poolman RW, van Hoogstraten MJ, et al. Immediate mobilization gives good results in boxer's fractures with volar angulation up to 70 degrees: a prospective randomized trial comparing immediate mobilization with cast immobilization. Arch Orthop Trauma Surg. 2003;123:534-537.

7. Ali A, Hamman J, Mass DP. The biomechanical effects of angulated boxer's fractures. J Hand Surg Am. 1999;24:835-844.

8. Braakman M, Oderwald EE, Haentjens MH. Functional taping of fractures of the 5th metacarpal results in a quicker recovery. Injury. 1998;29:5-9.

9. Zong SL, Zhao G, Su LX, et al. Treatments for the fifth metacarpal neck fractures: a network meta-analysis of randomized controlled trials. Medicine (Baltimore). 2016;95:e3059.

10. van Aaken J, Fusetti C, Lucchina S, et al. Erratum to: fifth metacarpal neck fractures treated with soft wrap/buddy taping compared to reduction and casting: results of a prospective, multicenter, randomized trial. Arch Orthop Trauma Surg. 2016;136:1481.

11. Hansen PB, Hansen TB. The treatment of fractures of the ring and little metacarpal necks. A prospective randomized study of three different types of treatment. J Hand Surg Br. 1998;23:245-247.

12. Kuokkanen HO, Mulari-Keranen SK, Niskanen RO, et al. Treatment of subcapital fractures of the fifth metacarpal bone: a prospective randomised comparison between functional treatment and reposition and splinting. Scand J Plast Reconstr Surg Hand Surg. 1999;33: 315-317.

13. McNemar TB, Howell JW, Chang E. Management of metacarpal fractures. J Hand Ther. 2003;16:143-151.

14. Dunn JC, Kusnezov N, Orr JD, et al. The boxer's fracture: splint immobilization is not necessary. Orthopedics. 2016;39:188-192.

15. Gamble D, Jenkins PJ, Edge MJ, et al. Satisfaction and functional outcome with self-care for the management of fifth metacarpal fractures. Hand (NY). 2015;10:607-612.

16. Bansal R, Craigen MA. Fifth metacarpal neck fractures: is follow-up required? J Hand Surg Eur Vol. 2007;32:69-73.

17. ten Berg PW, Ring D. Patients lost to follow-up after metacarpal fractures. J Hand Surg Am. 2012;37:42-46.

18. Institute for Work \& Health. The DASH outcome measure 2013. Available at: http://www.dash.iwh.on.ca. Accessed December 10, 2015.

19. London DA, Stepan JG, Boyer MI, et al. Performance characteristics of the verbal QuickDASH. J Hand Surg Am. 2014;39:100-107.

20. Stepan JG, London DA, Boyer MI, et al. Accuracy of patient recall of hand and elbow disability on the QuickDASH questionnaire over a two-year period. J Bone Joint Surg Am. 2013;95:e176.

21. EuroQol Research Foundation. EQ-5D 2017. Available at: https:// euroqol.org/eq-5d-instruments/eq-5d-3l-about/. Accessed December 12, 2015.

22. Viney R, Norman R, King MT, et al. Time trade-off derived EQ-5D weights for Australia. Value Health. 2011;14:928-936. 